\title{
An embryonic CaVß1 isoform promotes muscle mass maintenance via GDF5 signaling in
} adult mouse.

Authors: Traoré Massiré ${ }^{1 \dagger}$, Gentil Christel ${ }^{2 \dagger}$, Benedetto Chiara ${ }^{2}$, Hogrel Jean-Yves ${ }^{3}$, De la Grange Pierre ${ }^{4}$, Cadot Bruno ${ }^{2}$, Benkhelifa-Ziyyat Sofia ${ }^{2}$, Julien Laura $^{2}$, Lemaitre Mégane ${ }^{5}$, Ferry Arnaud $^{2}$, Piétri-Rouxel France ${ }^{2 * \#}$ and Falcone Sestina ${ }^{2 * \#}$

${ }^{1}$ Inovarion F-75013, Paris France.

${ }^{2}$ Sorbonne Université, Centre de Recherche en Myologie, UM76/INSERM U974 Institut de Myologie, F-75013, Paris, France.

${ }^{3}$ Institut de Myologie, GH Pitié-Salpêtrière, F-75013, Paris France;

${ }^{4}$ Genosplice, F-75005, Paris, France ;

${ }^{5}$ Sorbonne Université, INSERM, UMS28, F-75013, Paris, France

$\dagger:$ These authors equally contributed to this work.

\#: These authors equally contributed to this work.

*To whom correspondence should be addressed: Falcone Sestina: sestinaf@gmail.com;

Piétri-Rouxel France: france.pietri-rouxel@upmc.fr.

One sentence summary: $\mathrm{CaV} \beta 1 \mathrm{E}$ boosts down-stream Growth Differentiation Factor 5 signaling to counteract muscle mass loss in denervated or aged mouse muscles. 


\begin{abstract}
Deciphering the mechanisms that govern skeletal muscle plasticity is essential to understand its pathophysiological processes, including age-related sarcopenia. The voltage-gated calcium channel CaV1.1 has a central role in excitation-contraction coupling (ECC), raising the possibility that it may also initiate the adaptive response to changes during muscle activity. Here, we revealed the existence of a transcription switch of the CaV1.1 beta subunit $(\mathrm{CaV} \beta 1)$ gene that is dependent on the innervation state of the muscle in mice. In a mouse model of sciatic denervation we showed increased expression of an embryonic isoform of the subunit that we called CaV $\beta 1 \mathrm{E}$. CaV $\beta 1 \mathrm{E}$ boosts down-stream Growth Differentiation Factor 5 (GDF5) signaling to counteract muscle loss after denervation in mice. We further reported that aged mouse muscle expressed lower quantity of $\mathrm{CaV} \beta 1 \mathrm{E}$ compared to young muscle, displaying an altered GDF5dependent response to denervation. Conversely, $\mathrm{CaV} \beta 1 \mathrm{E}$ over-expression improved mass wasting in aging muscle in mice by increasing GDF5 expression. Crucially, we also identified the human $\mathrm{CaV} \beta 1 \mathrm{E}$ analogous and show a correlation between $\mathrm{CaV} \beta 1 \mathrm{E}$ expression in human muscles and age-related muscle mass decline. These results suggest that strategies targeting $\mathrm{CaV} \beta 1 \mathrm{E}$ or GDF5 might be effective in reducing muscle mass loss during senescence.
\end{abstract}

\title{
Introduction
}

A decrease in electrical activity, such as during neuromuscular disease, disuse, or aging can cause massive muscle atrophy (1). Disuse atrophy following muscle denervation or immobilization is characterized by the activation of a compensatory response to counteract mass loss. In particular, the induction of Growth Differentiation Factor 5 (GDF5)/SMAD4 (an acronym from the fusion of Caenorhabditis elegans Sma genes and the Drosophila Mad, Mothers against decapentaplegic) pathway is essential for avoiding excessive muscle mass loss but also for promoting reinnervation after nerve crush $(2,3)$. 
Aging muscles are characterized by progressive loss of mass and strength (4), suggesting an impairment of compensatory mechanisms. Currently, the best approaches to maintain aged muscle function and size are caloric restriction (limits autophagy and DNA damage) and exercise (restores muscle activity) (5-9). Yet, the evidence that muscle stimulation improves mass maintenance suggests that proteins sensing sarcolemmal depolarization might be suitable candidates for triggering a compensatory response. Among the five subunits of the L-type $\mathrm{Ca}^{2+}$ channel CaV1.1, CaVa1s subunit displays voltage sensor activity $(10,11)$. The intracellular subunit of the complex, CaV $\beta 1$ (encoded by Cacnbl gene), plays a role in (excitationcontraction coupling) ECC by targeting $\mathrm{CaV} \alpha 1 \mathrm{~s}$ to the membrane and regulating its activity (12). Currently, $\mathrm{CaV} \beta 1 \mathrm{~A}$ is considered as the skeletal muscle specific isoform (13). Furthermore, $\mathrm{CaV} \beta 1$ can be anchored to membrane, free in the cytoplasm, or located to nucleus of proliferating muscle cells where it acts as regulator of transcription factors (14). $\mathrm{CaV} \beta 1$ is essential for the development of embryonic mouse muscles and for the pre-patterning and development of neuromuscular junctions (NMJ), independently of ECC (14-16). Yet, no ECC-independent pathway involving $\mathrm{CaV} \beta 1$ is known in adult muscle.

Here, we provided the link between electrical activity sensing protein and muscle adaptation that compensates for atrophy. First, we demonstrated how the innervation state of skeletal muscle regulates the $\mathrm{CaV} \beta 1$ isoform transition epigenetically. We showed that $\mathrm{CaV} \beta 1 \mathrm{D}$ is the constitutively expressed isoform in adult mouse muscle, whereas $\mathrm{CaV} \beta 1 \mathrm{E}$ is the embryonic variant up-regulated upon impairment of electrical activity. Second, we highlighted CaV $\beta 1 \mathrm{E}$ as a major player in limiting muscle mass loss in mice through its ability to trigger GDF5 signaling in denervated muscle. In addition, we revealed that muscle aging is associated with altered $\mathrm{CaV} \beta 1 \mathrm{E} / \mathrm{GDF} 5$ axis and that over-expression of $\mathrm{CaV} \beta 1 \mathrm{E}$ drives the pathway necessary to 
counterbalance age-related muscle atrophy in rodents. Finally, we provided evidence of a human $\mathrm{hCaV} \beta 1 \mathrm{E}$ isoform expressed in adult muscle and tightly linked to muscle decline in aging.

\section{Results}

\section{Embryonic CaVß1E expression in adult mouse muscle}

Resection of mouse sciatic nerve is a suitable model for measuring the response of adult skeletal muscle to alterations in activity $(1,17)$. To establish if the expression of Cacnbl could change in this model we quantified its mRNA by RT-qPCR using primers in exons 2 and 3. We observed a time-dependent increase in the amplification of this region in denervated tibialis anterior muscles (TAs) compared to innervated control muscles (D0) (Fig 1A). Western blot of CaV $\beta 1$ revealed the appearance of an extra $70 \mathrm{kDa}$ band whose intensity increased with time after denervation while the intensity of the band around $55 \mathrm{kDa}$ was unchanged (Fig 1B, C).

The Cacnb1 gene (GSMG0007319) has 14 exons that can be spliced to give 6 transcript variants (Fig 1D). To identify potential Cacnbl splicing events occurring in denervated muscle, we performed a genome-wide transcriptomic analysis at the exon level on RNA extracted from innervated and denervated mouse TAs. We found 1022 differentially regulated alternative splicing events (from 706 distinct genes) (Datafile S1). Among these, Cacnb1 displayed a first exon splicing event showing that the transcript started in a putative non-coding sequence at the 5 ' end of exon 3 in innervated muscles. In denervated muscles, another transcript of Cacnbl starting at exon 1 was found to be upregulated (Fig 1E and Table S1), implying the transcription of two different splicing isoforms. RT-PCR confirmed that in innervated TAs the Cacnbl open reading frame is at the 5' end of exon 3 (ATG1). By contrast, in denervated muscles, two Cacnb1 transcripts were expressed: one starting at exon 1 (ATG2) and another at ATG1-exon 3 (Fig 1F, G). 
By searching Cacnb1 variants in the National Center for Biotechnology Information (NCBI) database, we found that Cacnb1-D is the only one starting immediately upstream the exon 3. Based on these results, our data suggest that the specific $\mathrm{CaV} \beta 1$ isoform expressed in the adult mouse skeletal muscle is $\mathrm{CaV} \beta 1 \mathrm{D}$ and not $\mathrm{CaV} \beta 1 \mathrm{~A}$, as expected (14). Moreover, the size of the extra CaV $\beta 1$ band appearing in denervated muscle suggested that it could be $\mathrm{CaV} \beta 1 \mathrm{~B}$ or $\mathrm{CaV} \beta 1 \mathrm{E}$ (66 and $70 \mathrm{kDa}$, respectively). However, $\mathrm{CaV} \beta 1$ antibody targeting a central peptide of the protein (AbCaVß1: sc-25689 (H-50) raised against amino acids 211-260 L-type CaVß1A of human origin - NM_199247-NP_954855) recognized only CaV $\beta 1 E$ in mouse. To validate which isoform was upregulated after denervation, we designed specific primers matching the two sequences at the level of the 3'-end of exon 13 for Cacnb1-D and exon 14 for Cacnb1-E. RTPCR data confirmed that only Cacnbl-E increased in denervated muscle (Fig 1H, I) confirming protein expression data.

Next, we verified if $\mathrm{CaV} \beta 1 \mathrm{E}$ was the only isoform induced in denervated muscle. Amplification of the region between exons 5 and 8/9 of Cacnb-1 let the discrimination of the several variants depending on the amplicon size. RT-PCR showed that innervated and denervated muscle expressed only Cacnb1 variants containing exon 7A, corresponding to Cacnb1-D and Cacnb1-E (381 bp), and not Cacnb1-B (246 bp), expressed in spinal cord (SC) (18-20) nor Cacnb1-C (246 bp) and Cacnbl-F (222 pb) (Fig 1J).

Several embryonic proteins are induced after muscle denervation $(3,21-25)$. We wondered whether CaV $\beta 1 \mathrm{E}$ could be an embryonic isoform. RT-PCR (primers matching exon 14) (Fig 1K) and RT-qPCR (primers in exons 2-3 for Cacnb1-E and exon 13 for Cacnb1-D) revealed that Cacnbl-E is the specific variant in embryonic and neonatal muscles, while Cacnbl-D is not expressed in embryo (Fig S1A, B). Cacnb1-E transcript in embryonic muscle started at exon 1 (Fig S1C, D) as Cacnbl-E expressed in denervated adult muscle. Western blotting showed the 
same bands in denervated adult and embryonic muscle protein extracts using AbCaV $\beta 1$ (Fig 1L) or an antibody specific to CaV $\beta 1 \mathrm{E}$ (Fig S1E). Finally, we analyzed if CaV $\beta 1 \mathrm{D}$ and $\mathrm{CaV} \beta 1 \mathrm{E}$ could have different localization in muscle fiber. Immunofluorescence analysis of innervated and denervated muscle sections and isolated fibers with $\mathrm{AbCaV} \beta 1$ showed that $\mathrm{CaV} \beta 1$ staining and triadic localization most likely reflected expression of CaV $\beta 1 \mathrm{D}$ (Fig S2A-C). In contrast, CaV $\beta 1$ E-specific staining was distributed at the Z-lines (Fig S2 D-F). Furthermore, CaV $\beta 1 E-$ specific staining localized mostly at the nuclei, consistently with the presence of a Nuclear Localization Signal predicted by cNLS mapper, and its intensity increased in denervated muscle compared to $\mathrm{CaV} \beta 1$ staining (Fig S2G-L).

In conclusion, our results show that an alternative first exon splicing is the source of the differential expression of mouse adult and embryonic Cacnbl variants. $\mathrm{CaV} \beta 1 \mathrm{D}$, not $\mathrm{CaV} \beta 1 \mathrm{~A}$, is expressed in innervated adult skeletal muscle, whereas embryonic muscle expresses CaV $\beta 1 \mathrm{E}$. $\mathrm{CaV} \beta 1 \mathrm{E}$ is low in innervated adult mouse muscle but denervation specifically increases its expression. Moreover, $\mathrm{CaV} \beta 1 \mathrm{D}$ and $\mathrm{CaV} \beta 1 \mathrm{E}$ display different intracellular locations in adult muscle fibers.

\section{CaVB1E and GDF5 signaling activation after denervation}

To evaluate whether $\mathrm{CaV} \beta 1 \mathrm{E}$ has a role in disuse atrophy, we injected into mouse $\mathrm{TA}$ an Associated-adeno-virus (AAV) vector carrying a short-hairpin RNA targeting a sequence in Cacnb1 exon 2 (26) (AAV-ShCaV $\beta 1 E$ ) and thereby abolishing specifically Cacnb1-E. Two months after injection, $\mathrm{CaV} \beta 1 \mathrm{E}$ expression induced after denervation was decreased by $\sim 90 \%$ (Fig 2A-C; Fig S3A, B), without decreasing CaV $\beta 1$ D protein expression (Fig 2B, C). Blocking the induction of $\mathrm{CaV} \beta 1 \mathrm{E}$ exacerbated muscle atrophy after denervation (Fig 2D-F), with decreased muscle fiber size (Fig 2F) and increased fibrosis (Fig S3C-F). This suggested a role for $\mathrm{CaV} \beta 1 \mathrm{E}$ in preserving disused muscle mass. The GDF5 pathway has been shown to be essential 
for limiting muscle loss under atrophic conditions (2). We thus evaluated Gdf5 expression in absence of $\mathrm{CaV} \beta 1 \mathrm{E}$ and found a significant $(\mathrm{P}<0.0001)$ reduction in the denervation-induced Gdf5 increase (Fig 2G). In contrast, Gdf8 or Bmp7 transcription, which could be both implicated in increased atrophy $(27,28)$, was not modified upon CaV $\beta 1 \mathrm{E}$ ablation (Fig 2H, I). Furthermore, all components of the GDF5 pathway, SMAD1/5 phosphorylation, SMAD4 nuclear translocation, and $I d-2$ transcription $(29,30)$, were inhibited with $\mathrm{CaV} \beta 1 \mathrm{E}$ down-regulation suggesting the positive control of GDF5 signaling by CaV $\beta 1 \mathrm{E}$ (Fig 2J-O). However, Id-1 expression was not modified after denervation in Scra or AAV-ShCaV $\beta 1 \mathrm{E}$ treated muscle (Fig $2 \mathrm{~N})$, suggesting that its activation might be very weak or transient (31).

$\mathrm{CaV} \beta 1$ has been shown to inhibit myogenin signaling during myoblast proliferation (14), yet there are no data about this regulation in adult muscle. In innervated or denervated TAs we found no change in transcription of Myogenin after CaV $\beta 1 \mathrm{E}$ knock-down (Fig S3G) and no alterations in Fbxo32, MuRF1 expression (Fig S3H-I), indicating that the myogenin pathway is unlikely to participate in the increased atrophy following $\mathrm{CaV} \beta 1 \mathrm{E}$ down-regulation. However, Chrna 1 transcription after denervation was significantly $(\mathrm{P}<0.0001)$ reduced in absence of $\mathrm{CaV} \beta 1 \mathrm{E}$, suggesting its involvement in modulating genes associated with endplate formation, independently of myogenin. A previous study showed that in proliferating muscle cells canonical and non-canonical DNA E-box sequences (CANNTG and CANNNTG) of several promoter regions could be targeted by $\mathrm{CaV} \beta 1$ (14). Analysis of the 100 most differentially regulated genes in innervated and denervated TA revealed the presence of one or more E-box sequences in the promoter of all these genes (Table S2). However, among them, only Gdf5 and myogenin had a demonstrable role in muscle mass homeostasis $(31,32)$.

To further analyze the role of $\mathrm{CaV} \beta 1 \mathrm{E}$ on $G d f 5$ expression, we used the myogenic cell line $\mathrm{C} 2 \mathrm{C} 12$ as tool in vitro. $\mathrm{C} 2 \mathrm{C} 12$ cells expressed increasing Cacnb1-E during differentiation (Fig 
$\mathrm{S} 4 \mathrm{~A}, \mathrm{~B}$ ) and only CaV $\beta 1 \mathrm{E}$ protein (band at 70kDa) as embryonic muscle. (Fig S4C). Moreover, the transcription of Gdf5, as well as Myogenin, also increased in differentiating C2C12 cells (Fig S4D, E). Inhibiting Cacnb1-E expression in differentiating C2C12 cells by transfecting a plasmid ShCaV $\beta 1 \mathrm{E}$ (pCDNA3-ShCaV $\beta 1 \mathrm{E}$ ) (Fig S4F) prevented the expression of $G d f 5$ without affecting Myogenin transcription (Fig S4G, H), mimicking its effect in vivo. We then measured in $\mathrm{C} 2 \mathrm{C} 12$ the consequence of $\mathrm{CaV} \beta 1 \mathrm{E}$ down-regulation on the promoter activity of $G d f 5$. Thus, the sequence from -312 to the $G d f 5$ transcription start site, which contains one CANNTG and two CANNNTG E-boxes, was cloned upstream of firefly luciferase in HSVTK-Luc3' modified plasmid and transfected into $\mathrm{C} 2 \mathrm{C} 12$ cells. Firefly/renilla signal increased during differentiation, reflecting $G d f 5$ promoter activation, and this effect was abolished by the ShCaV $\beta 1 \mathrm{E}$ (Fig 2P). These data indicated that $\mathrm{CaV} \beta 1 \mathrm{E}$ targets $G d f 5$ promoter, consistently with the effect observed in vivo and in vitro.

$\mathrm{CaV} \beta 1 \mathrm{E}$ seems to precede $\mathrm{CaV} \beta 1 \mathrm{D}$ appearance in embryo and in $\mathrm{C} 2 \mathrm{C} 12$. We thus checked if Cacnb1E and Cacnb1D mRNA expression could affect each other and have a role in muscle mass homeostasis via GDF5. Although AAV-ShCaVß1E injection prevented Cacnb1E rise after denervation, it also induced a slight decrease of Cacnb1D mRNA expression (Fig S5A, B), yet with no impact on $\mathrm{CaV} \beta 1 \mathrm{D}$ protein expression. However, only the rescue of $\mathrm{CaV} \beta 1 \mathrm{E}$, and not $\mathrm{CaV} \beta 1 \mathrm{D}$, restored $G d f 5$ transcription and atrophy after denervation in AAV-ShCaV $\beta 1 \mathrm{E}$ treated muscles (Fig S5C, D).

To confirm that the increased atrophy upon $\mathrm{CaV} \beta 1 \mathrm{E}$ down-regulation was dependent on inadequate GDF5 activation, we overexpressed this factor in TAs together with ShCaV $\beta 1 \mathrm{E}$, via AAV-gene transfer (Fig S5E, F). The up-regulation of $I d 1$ and $I d 2$ proved the activation of GDF5-dependent pathway (fig S5G, H) in double treated muscles. As expected, GDF5 over- 
expression rescued the atrophy induced $\mathrm{CaV} \beta 1 \mathrm{E}$ ablation and improved muscle histology by decreasing fibrosis (Fig S5I-R).

\section{Aging muscles: a key role for CaVß1E}

During aging skeletal muscle shows denervation-like signs and progressive muscle wasting (4, 33). This suggests that impairment in compensatory response might occur. However, little is known about CaV $\beta 1$ expression and function (26) and nothing is known about $G d f 5$ amount in aging muscle.

Because our data indicate the involvement of $\mathrm{CaV} \beta 1 \mathrm{E}$ via $G d f 5$ in muscle maintenance, we investigated its role in age-related muscle wasting. In C57bl/6 mice, we observed significant $(\mathrm{P}<0.0001)$ TA muscle mass loss at 78 weeks relative to adult mice at 12 weeks (Fig $3 \mathrm{~A})$. In addition, Cacnb1-E basal expression was significantly lower in TAs of 78 compared to 12 weekold mice ( $\mathrm{P}<0.0001)$ (Fig 3B), whereas Cacnb1-D transcription did not change (Fig 3C). To evaluate if the cross-talk between Cacnb1-E and Gdf5 would also be affected, we quantified these transcripts after denervation in TAs from 12-, 52- and 78-week-old mice. Although CaV $\beta 1 \mathrm{E}$ expression increased in denervated young muscle, the up-regulation of transcript and protein in response to denervation was impaired since 52 weeks of age (Fig 3D, G, H). Consequently, the Gdf5 increase was reduced (Fig 3F) affecting SMAD1/5 phosphorylation (Fig 3J, K). Id1

remained unchanged (Fig 3L) whereas $I d-2$ transcription followed the altered $G d f 5$ induction (Fig 3M). CaV $\beta 1 \mathrm{D}$ expression did not change (Fig 3E, G, I).

We explored some possible causes responsible for decreasing $\mathrm{CaV} \beta 1 \mathrm{E}$ during aging. Measuring the expression of NMJ components Chrnal, Chrne, Chrng and Musk in 78 week-old mice muscles, we could not detect changes indicating endplate alterations at this age (Fig 4A-D). Similarly, we checked if age-related modifications in fiber type composition occurred, maybe explaining $\mathrm{CaV} \beta 1 \mathrm{E}$ down-regulation. Expression of myosin heavy chain (MyHC) isotypes IIA 
and IIX decreased significantly during aging $(\mathrm{P}=0.00616652$ and $\mathrm{P}=0.01394505$, respectively), as reported $(9,34,35)$, whereas $M y H C$-I and -IIB did not change between 12 and 78 weeks of age (Fig 4E-H). To elucidate if $\mathrm{CaV} \beta 1 \mathrm{E}$ expression could be associated to fiber type modifications, we analyzed its amount in MyHC-I and -II positive fibers by immunofluorescence. In young muscle, $\mathrm{CaV} \beta 1 \mathrm{E}$ was expressed at higher intensity in MyHCIIA and -IIX positive fibers, although in old muscle it was decreased in all fiber types (Fig 4I$\mathrm{K})$.

If $\mathrm{CaV} \beta 1 \mathrm{E}$ increased upon lack of nerve activity, for example denervation, modifications in muscle activity could potentially affect basal CacnblE transcription. Hence, we measured CacnblE mRNA after acute exercise of young and old mice $(36,37)$. Exercise training restored Cacnb1E transcription in old muscles but not in young (Fig 4L), with Cacnb1D expression unaffected (Fig 4M), suggesting that increased muscle activity is able to rapidly normalize CacnblE transcript only when it is decreased. However, MyHC-IIA and IIX expressions were not restored, implying that contractile activity could regulate CacnblE expression independently of type IIA-X fiber abundance (Fig 4N, O).

To evaluate whether CaV $\beta 1 \mathrm{E}$ or GDF5 over-expression might mitigate age-related muscle mass loss, AAV vectors carrying CaV $\beta 1 \mathrm{E}$ or GDF5 were injected into the TA of 78-80-week-old mice. A strong over-expression of $\mathrm{CaV} \beta 1 \mathrm{E}$ and GDF5 was observed 3 months after injection (Fig. 5A, B, D and S6A) without modifying Cacnb1-D expression (Fig 5C and S6C), yet increasing expression of each other reciprocally (Fig 5D and S6B). The up-regulation of Cacnb1E in old muscle activated GDF5 signaling, as measured by SMAD1/5 phosphorylation and SMAD4 nuclear translocation. (Fig 5E-G). The increase of $G d f 5$ either by CaV $\beta 1 \mathrm{E}$ or by its own over-expression induced $I d$-2 transcription, with no changes in $I d-1$, confirming a weaker or transient response of this factor to GDF5 (Fig 5H, I and S6D, E). In addition, the rise of GDF5 
signaling following Cacnbl-E or Gdf5 over-expression was associated with preservation of aged muscle mass (Fig 5J) and a gain of specific force (Fig 5K) compared to control old muscles. Subsequently, we investigated if $\mathrm{CaV} \beta 1 \mathrm{E}$ over-expression could affect muscle mass of young mice (12 weeks). Ectopic expression of CaV $\beta 1 \mathrm{E}$ (Fig S7A, D, E) had no effect on Cacnb1-D, yet it induced a slight increase of Gdf5 expression (Fig S7B, C, D, E). However GDF5 signaling, measured by $I d-1$ and $I d-2$ transcription, was not or poorly activated (Fig S7F, G). Consequently, muscle mass and the response to denervation were not different between Scra- and CaV $\beta 1 \mathrm{E}-$ treated TAs of young mice (Fig S7H-L). We also overexpressed GDF5 in young TAs (Fig S8A, D, E) which induced Cacnbl-E transcription in innervated TAs compared to scrambled (Fig S8B, D, E) without affecting Cacnb1-D expression (Fig S8C). Nevertheless, GDF5 over-expression and its activated signaling, measured as $I d-1$ and $I d-2$ transcription (Fig S8F, G), increased mostly innervated muscle mass (Fig S8H-L).

Overall, these data show that the loss of $\mathrm{CaV} \beta 1 \mathrm{E} / \mathrm{GDF} 5$ cross-talk observed during aging may be critical for muscle wasting and its rescue counteracts the process of age-related muscle decline.

\section{Human muscle: a new CaV $\beta E$ isoform implicated in skeletal muscle aging}

Given the apparent importance of $\mathrm{CaV} \beta 1 \mathrm{E}$ in mouse skeletal muscle, we wondered whether an analogous mechanism might be conserved in humans and, thus, if another unidentified $C A C N B 1$ isoform was expressed in adult human skeletal muscle. Three human CACNB1 (hCACNB1) variants have been identified corresponding to the mouse isoforms A (specific of skeletal muscle), B, and C (Fig 6A) (18-20,38). Human mRNA extracted from one quadriceps and two fascia lata (FL) muscle biopsies of healthy adult subjects together with human mRNA extracted from the cervical spinal cord (Table S2), as positive control for hCACNB1-B (18-20), were probed for exons 13 and 5-9. Amplification of the sequence in exon 13 showed that all muscles expressed hCACNB1-A. As in mouse muscle, amplification of the region between exons 5 and 9 
demonstrated that only a $380 \mathrm{bp}$ corresponding to the putative hCACNB1-A appeared and that the exon 7B-containing isoform, hCACNB1-B (245 bp), was solely expressed in spinal cord (SC). Furthermore, no transcription of $\mathrm{h} C A C N B 1-\mathrm{C}$, which also has the short $7 \mathrm{~B}$ exon, was found in muscle (Fig 6B). We further checked if human muscle could express a hCACNB1 transcript analogous to mouse Cacnb1-E. Amplification of the region in exon 14 revealed that the human muscles expressed the previously unidentified variant that we have called hCACNB1-E (Fig 6C). This isoform corresponded to the predicted XM_006722072.2 variant, having a codon start (ATG2) upstream the exon 3 (Fig 6A, C). Western blot and immunofluorescence experiments confirmed its expression in two different human FLs (Fig 6D, E), with localization similar to that of mouse $\mathrm{CaV} \beta 1 \mathrm{E}$ (Fig S2E, F).

Since we found that altered $\mathrm{CaV} \beta 1 \mathrm{E} / \mathrm{GDF} 5$ axis was associated to muscle wasting during aging in mice, we compared characteristics indicating muscle mass (lean mass percentage) and function (power) $(39,40)$ in a cohort of healthy young (20-42 years) and aged (70-81 years) volunteers, included in a previous study (40) (Table 1). Aged group displayed significantly lower lean mass and power than young group $(\mathrm{P}=0.003255$ and $\mathrm{P}<0.0001$, respectively) (Fig $6 \mathrm{~F}, \mathrm{G})$. We then measured $\mathrm{hCACNB}$ 1-E transcript and found a significant reduction $(\mathrm{P}=0.0045)$ of its expression in aged group (Fig 6H), whereas hCACNB1-A transcription did not differ between groups (Fig 6I). A low hCACNB1-E expression was also associated with a low lean mass percentage (Fig 6J). We detected a weak amount of hGDF5 in muscle biopsies, however, in aged muscle samples we could associate low expression of both hCACNB1-E and hGDF5 with low lean mass percentage. In addition, participants with higher lean mass percentage displayed higher expression of both hCACNB1-E and hGDF5 (Fig 6K).

Overall these results suggest that $\mathrm{CaV} \beta 1 \mathrm{E} / \mathrm{GDF} 5$ axis in compensatory response and aging (Fig S9) might be a conserved mechanism between mice and humans. 


\section{Discussion}

In adult skeletal muscle, the mechanism connecting electrical activity sensing and changes in gene expression is unclear. Here, we showed that denervation boosts the expression of embryonic $\mathrm{CaV} \beta 1 \mathrm{E}$ isoform which mediates transcriptional reprogramming in adult muscle. Many years ago CaV $\beta 1$ isoforms were identified in humans $(19,20,41)$, rat (18) and, less clearly, in mouse (13). We anticipated that $55 \mathrm{kDa}$ band corresponded to $\mathrm{CaV} \beta 1 \mathrm{~A}$, a previously identified musclespecific isoform with this molecular weight $(18,19)$. However, we speculated that the $70 \mathrm{kDa}$ band could be a previously unappreciated $\mathrm{CaV} \beta 1$ isoform. Our results revealed that $\mathrm{CaV} \beta 1 \mathrm{D}$, not $\mathrm{CaV} \beta 1 \mathrm{~A}$, is the main constitutive isoform specific to normal adult mouse muscle and that $\mathrm{CaV} \beta 1 \mathrm{D}$ and $\mathrm{CaV} \beta 1 \mathrm{E}$ have distinct roles. Indeed, in silico analysis using cNLS mapper confirmed the presence of a Nuclear Localization Signal at the N-terminus of CaV $\beta 1 \mathrm{E}$ but not in $\mathrm{CaV} \beta 1 \mathrm{D}$. CaV $\beta \mathrm{A}$ is not expressed in adult mouse muscle and $\mathrm{C} 2 \mathrm{C} 12$, however, its $\mathrm{N}$-terminus is putatively identical with those of $\mathrm{CaV} \beta \mathrm{E}$, suggesting that the mechanism by which it translocates to the nucleus is the same described in past publications $(14,42)$. This finding also implicates $\mathrm{CaV} \beta \mathrm{D}$ in regulating calcium channel assembly in the cytoplasm, whereas CaV $\beta \mathrm{E}$ could have a role in both modulating nuclear transcription factors and cooperating with cytoskeletal or Ttubules at the Z-Lines, as demonstrated for others proteins $(43,44)$.

We also discovered that $\mathrm{CaV} \beta 1 \mathrm{E}$ is the main $\mathrm{CaV} \beta 1$ isoform in proliferating and differentiating C2C12 cells, while CaV $\beta 1 \mathrm{D}$ and $\mathrm{CaV} \beta 1 \mathrm{~A}$ isoforms are undetectable. Previous studies reporting expression, transcriptional activity and nuclear localization of $\mathrm{CaV} \beta 1 \mathrm{~A}$ in muscle fibers (42), muscle precursors and $\mathrm{C} 2 \mathrm{C} 12$ cells (14) were likely evaluating the as-yet undiscovered $\mathrm{CaV} \beta 1 \mathrm{E}$. An interesting issue of our study could be deciphering mechanisms underlying the transition 
between $\mathrm{CaV} \beta 1 \mathrm{E}$ and $\mathrm{CaV} \beta 1 \mathrm{D}$ during myogenesis and identifying molecular factors regulating the implicated splicing events.

The major finding of this work is the central role of $\mathrm{CaV} \beta 1 \mathrm{E}$ in maintaining muscle mass. GDF5 is one of the main regulators of muscle mass homeostasis in response to disuse atrophy (31), however few studies report progress on modulators of this factor $(3,45,46)$. Our data validate previously described roles of $\operatorname{GDF} 5(31,47)$ and add a positive upstream player controlling its signaling. Indeed, $\mathrm{CaV} \beta 1 \mathrm{E}$ expression is needed for the activation of $G d f 5$ promoter, probably by acting on specific E-box sequences as described for other target genes (14).

In addition, we demonstrated that a compromised CaV $\beta 1 \mathrm{E} / \mathrm{GDF} 5$ axis during aging is associated to muscle wasting. In our study, $\mathrm{CaV} \beta 1 \mathrm{E}$ over-expression led to increased GDF5 expression and activation of its pathway improving age-related muscle decline. By contrast, $\mathrm{CaV} \beta 1 \mathrm{E}$ overexpression did not affect muscle mass in young mice. This suggests that endogenous $\mathrm{CaV} \beta 1 \mathrm{E}$ induction following nerve withdrawal is sufficient to trigger the maximal compensatory response. In addition, when muscles express physiological amount of GDF5, feedback mechanisms could be activated to prevent hypertrophy. Interestingly, GDF5 exerted hypertrophic effects mostly on innervated muscle, suggesting that nerve is important for its trophic signal. In this hypothesis, GDF5 could stimulate pre- and/or post-synaptic NMJ partners improving neuro-transmission. Thus, increased GDF5 signaling, induced by over-expression of CaV $\beta 1 \mathrm{E}$ or GDF5 itself, might counteract age-related muscle decline also by preventing the associated denervation occurring later. $\mathrm{CaV} \beta 1$ has been shown as important for correct synaptic patterning in embryo (15). Even though a role for GDF5 in this process has not been established yet, its relevance to mediate reinnervation in adult muscle is proven (3). Importantly, we also demonstrated that a hCACNB1-E variant is expressed in human muscle, indicating that mechanisms governing skeletal muscle homeostasis might be conserved across mammalian species. Although hCACNB1-E transcript is 
slightly different compared to mouse Cacnbl-E, we observed similar localization and hypothesize the same function. In fact, $\mathrm{h} C A C N B 1-\mathrm{E}$ expression was higher in young compared to aged human muscle with reduced lean mass and power, strongly suggesting a defect in compensatory mechanism contributing to age-related muscle mass loss. Despite weak transcription of hGDF5 in undamaged young human muscle, we demonstrated an association between its expression and muscle mass.

Other studies identified hypertrophic and potentially druggable factors reduced in sarcopenic muscle as IGF-1 (7), GH (48), leptin (49) and, recently, apelin (9). These molecules are dependent on muscle activity and aging. Here, we identified CaV $\beta 1 \mathrm{E}$ as "sensor" of electrical activity defect which could be a central player, upstream of all these factors. Age-related muscle decline is a life-threatening condition (50) affecting a large population, which results in progressive loss of autonomy, increased mortality associated to frailty and risk of falls in elderly subjects (51). GDF5 or GDF5-like molecules can be considered as potential therapeutic compounds to prevent muscle mass wasting during senescence but also, in a wider application, to ameliorate consequences of neuromuscular defects. Finally, deciphering the fine regulation of CaV $\beta 1 \mathrm{E}$ expression and GDF5 signaling would open a promising therapeutic field that will contribute to increasing the quality of life.

The study has some limitations. The events altering the correct basal expression of $\mathrm{CaV} \beta 1 \mathrm{E}$ in aging muscle remain unresolved. Previous studies have reported a link between elevated $\mathrm{CaV} \beta 1 \mathrm{~A}$ and age-related muscle weakness $(26,52,53)$ but Cacnb1 transcripts were not fully characterized nor antibodies used well defined. CaV $\beta 1 \mathrm{E}$ expression is higher in young type IIAIIX fibers compared to IIB. The fiber type II decrease occurring during aging may thus have a role in $\mathrm{CaV} \beta 1 \mathrm{E}$ reduction. Still, other mechanisms, dependent on muscle activity per se, 
modifying DNA methylation or mitochondrial metabolism, could participate in altering the $\mathrm{CaV} \beta 1 \mathrm{E} / \mathrm{GDF} 5$ axis.

\section{Materials and Methods}

\section{Study design}

The primary objective of this study was to decipher the link between alteration of electrical activity and skeletal muscle mass maintenance. We focused on the role of CaV $\beta 1$, a subunit of the voltage sensor CaV1.1. RNAseq data of innervated and denervated TAs led to identify an embryonic variant, $\mathrm{CaV} \beta 1 \mathrm{E}$, up-regulated in mouse muscle by denervation. Validation of RNAseq data and characterization of $\mathrm{CaV} \beta 1$ isoforms in mouse and human muscles have been performed by RT-PCR, qPCR, western blot and immunostaining experiments. To investigate the role of $\mathrm{CaV} \beta 1 \mathrm{E}$ in muscle mass homeostasis in mouse, we knocked-down its local expression in vivo by AAV-shRNA. We quantified atrophy by measuring muscle weight and fiber size distribution. To determine fiber size, images were processed with a machine learning algorithm (WEKA) under Fiji for accurate segmentation. Muscle histology was performed by Haematoxilin\&Eosin and Sirius Red staining. To evaluate the effect of CaV $\beta 1 \mathrm{E}$ down-regulation on the transcriptional activity of $G d f 5$ promoter, we performed a Luciferase reporter-based assay in $\mathrm{C} 2 \mathrm{C} 12$. To establish the effect of $\mathrm{CaV} \beta 1 \mathrm{E}$ or GDF5 in vivo on muscle mass, we overexpressed them in mouse by local AAV-gene transfer. In order to characterize the expression of $\mathrm{CaV} \beta 1$ isoforms in humans, we used muscle biopsies of a cohort of healthy young and aged volunteers (40). Body composition and muscle function of volunteers included in the study (40) had been measured previously and provided for correlation analysis with hCACNB1-E. We estimated the sample size on the basis of known variability of assays. All mouse experiments were performed twice. Outliers not following normal distribution of samples were removed based 
on Grubb's test. All experiments using animals and cells were done in a non-blinded manner, yet investigators were blinded to allocation in using human samples for RT-qPCR experiments.

\section{Statistical Analysis}

For comparison between two groups, two-tailed paired and unpaired Student's $t$-tests were performed to calculate $\mathrm{P}$ values and to determine statistically significant differences

(significance was for $\mathrm{P}<0.05$, as detailed in the figure legends). For comparison among more than two groups, ordinary one or two-way Anova tests followed by the appropriated multiple comparison tests (as detailed in the figure legends) were performed. All experiments have been done twice with the same results. All statistical analyses were performed with GraphPad Prism7 software.

\section{Supplementary material list}

\section{Materials and Methods}

Figure S1: expression of embryonic CaVß1 isoform

Figure S2: CaVß1 isoforms localization on adult skeletal muscle

Figure S3: Histological characterization and myogenin signaling in CaVß1E knock-down muscles

Figure S4: Expression of Cacnb1-E, myogenin and Gdf5 in $\mathrm{C2C12}$

Figure S5: Effects of CaVß1E, CaVß1D and GDF5 over-expression on AAV-ShCaVß1E treated muscles

Figure S6: GDF5 over-expression in aged mice muscle

Figure S7: CaVß1E over-expression in young mice muscle

Figure S8: GDF5 over-expression in young mice muscle

Fig S9: Schematic representation of CaVß1E/axis in young and old mouse muscle Table S1: Expression data 


\section{Table S2: 100 top up-regulated genes in innervated and denervated samples}

\section{Table S3: List of human samples}

\section{Table S4: Primer list}

\section{Datafile S1: List of 1022 differentially regulated alternative splicing events}

\section{Datafile S2: Uncut Western Blotting raw data}

\section{Datafile S3: Individual subject data}

\section{References}

1. K. Gundersen, Excitation-transcription coupling in skeletal muscle: the molecular pathways of exercise. Biol Rev Camb Philos Soc. 86, 564-600 (2011).

2. R. Sartori, E. Schirwis, B. Blaauw, S. Bortolanza, J. Zhao, E. Enzo, A. Stantzou, E. Mouisel, L. Toniolo, A. Ferry, S. Stricker, A. L. Goldberg, S. Dupont, S. Piccolo, H. Amthor, M. Sandri, BMP signaling controls muscle mass. Nat Genet. 45, 1309-1318 (2013).

3. P. C. D. Macpherson, P. Farshi, D. Goldman, Dach2-Hdac9 signaling regulates reinnervation of muscle endplates. Development. 142, 4038-4048 (2015).

4. A. Pannérec, M. Springer, E. Migliavacca, A. Ireland, M. Piasecki, S. Karaz, G. Jacot, S. Métairon, E. Danenberg, F. Raymond, P. Descombes, J. S. McPhee, J. N. Feige, A robust neuromuscular system protects rat and human skeletal muscle from sarcopenia. Aging (Albany NY). 8, 712-729 (2016).

5. G. Valdez, J. C. Tapia, H. Kang, G. D. Clemenson, F. H. Gage, J. W. Lichtman, J. R. Sanes, Attenuation of age-related changes in mouse neuromuscular synapses by caloric restriction and exercise. Proc. Natl. Acad. Sci. U.S.A. 107, 14863-14868 (2010).

6. H. Kern, C. Hofer, S. Loefler, S. Zampieri, P. Gargiulo, A. Baba, A. Marcante, F. Piccione, A. Pond, U. Carraro, Atrophy, ultra-structural disorders, severe atrophy and degeneration of denervated human muscle in SCI and Aging. Implications for their recovery by Functional Electrical Stimulation, updated 2017. Neurol. Res. 39, 660-666 (2017).

7. H. Kern, L. Barberi, S. Löfler, S. Sbardella, S. Burggraf, H. Fruhmann, U. Carraro, S. Mosole, N. Sarabon, M. Vogelauer, W. Mayr, M. Krenn, J. Cvecka, V. Romanello, L. Pietrangelo, F. Protasi, M. Sandri, S. Zampieri, A. Musaro, Electrical stimulation counteracts muscle decline in seniors. Front Aging Neurosci. 6, 189 (2014).

8. S. Mosole, U. Carraro, H. Kern, S. Loefler, H. Fruhmann, M. Vogelauer, S. Burggraf, W. Mayr, M. Krenn, T. Paternostro-Sluga, D. Hamar, J. Cvecka, M. Sedliak, V. Tirpakova, N. Sarabon, A. Musarò, M. Sandri, F. Protasi, A. Nori, A. Pond, S. Zampieri, Long-term high-level exercise promotes muscle reinnervation with age. J. Neuropathol. Exp. Neurol. 73, 284-294 (2014).

9. C. Vinel, L. Lukjanenko, A. Batut, S. Deleruyelle, J.-P. Pradère, S. L. Gonidec, A. Dortignac, N. Geoffre, O. Pereira, S. Karaz, U. Lee, M. Camus, K. Chaoui, E. Mouisel, A. Bigot, V. Mouly, M. Vigneau, A. F. Pagano, A. Chopard, F. Pillard, S. Guyonnet, M. Cesari, O. Burlet-Schiltz, M. Pahor, J. N. Feige, B. Vellas, P. Valet, C. Dray, The exerkine apelin reverses age-associated sarcopenia. Nature Medicine, 1 (2018).

10. J. Schredelseker, V. Di Biase, G. J. Obermair, E. T. Felder, B. E. Flucher, C. Franzini-Armstrong, M. Grabner, The beta 1a subunit is essential for the assembly of dihydropyridine-receptor arrays in skeletal muscle. Proc. Natl. Acad. Sci. U.S.A. 102, 17219-17224 (2005). 
11. A. Dayal, J. Schredelseker, C. Franzini-Armstrong, M. Grabner, Skeletal muscle excitation-contraction coupling is independent of a conserved heptad repeat motif in the C-terminus of the DHPRbeta(1a) subunit. Cell Calcium. 47, 500-506 (2010).

12. A. Dayal, V. Bhat, C. Franzini-Armstrong, M. Grabner, Domain cooperativity in the $\beta 1$ a subunit is essential for dihydropyridine receptor voltage sensing in skeletal muscle. Proc. Natl. Acad. Sci. U.S.A. 110, 7488-7493 (2013).

13. R. G. Gregg, A. Messing, C. Strube, M. Beurg, R. Moss, M. Behan, M. Sukhareva, S. Haynes, J. A. Powell, R. Coronado, P. A. Powers, Absence of the $\beta$ subunit (cchb1) of the skeletal muscle dihydropyridine receptor alters expression of the $\alpha 1$ subunit and eliminates excitation-contraction coupling. PNAS. 93, 13961-13966 (1996).

14. J. Taylor, A. Pereyra, T. Zhang, M. L. Messi, Z.-M. Wang, C. Hereñú, P.-F. Kuan, O. Delbono, The Cavß1a subunit regulates gene expression and suppresses myogenin in muscle progenitor cells. J. Cell Biol. 205, 829846 (2014).

15. F. Chen, Y. Liu, Y. Sugiura, P. D. Allen, R. G. Gregg, W. Lin, Neuromuscular synaptic patterning requires the function of skeletal muscle dihydropyridine receptors. Nat Neurosci. 14, 570-577 (2011).

16. A. Dayal, K. Schrötter, Y. Pan, K. Föhr, W. Melzer, M. Grabner, The Ca2+ influx through the mammalian skeletal muscle dihydropyridine receptor is irrelevant for muscle performance. Nat Commun. 8, 475 (2017).

17. A. Méjat, F. Ramond, R. Bassel-Duby, S. Khochbin, E. N. Olson, L. Schaeffer, Histone deacetylase 9 couples neuronal activity to muscle chromatin acetylation and gene expression. Nat. Neurosci. 8, 313-321 (2005).

18. M. Pragnell, J. Sakamoto, S. D. Jay, K. P. Campbell, Cloning and tissue-specific expression of the brain calcium channel $\beta$-subunit. FEBS Letters. 291, 253-258 (1991).

19. P. A. Powers, S. Liu, K. Hogan, R. G. Gregg, Skeletal muscle and brain isoforms of a beta-subunit of human voltage-dependent calcium channels are encoded by a single gene. J. Biol. Chem. 267, 22967-22972 (1992).

20. K. Hogan, R. G. Greg, P. A. Powers, Structure and alternative splicing of the gene encoding the human beta1 subunit of voltage dependent calcium channels. Neurosci. Lett. 277, 111-114 (1999).

21. H. Tang, D. Goldman, Activity-dependent gene regulation in skeletal muscle is mediated by a histone deacetylase (HDAC)-Dach2-myogenin signal transduction cascade. Proc. Natl. Acad. Sci. U.S.A. 103, 1697716982 (2006).

22. L. Adams, B. M. Carlson, L. Henderson, D. Goldman, Adaptation of nicotinic acetylcholine receptor, myogenin, and MRF4 gene expression to long-term muscle denervation. J Cell Biol. 131, 1341-1349 (1995).

23. H. Tang, P. Macpherson, M. Marvin, E. Meadows, W. H. Klein, X.-J. Yang, D. Goldman, A histone deacetylase 4/myogenin positive feedback loop coordinates denervation-dependent gene induction and suppression. Mol. Biol. Cell. 20, 1120-1131 (2009).

24. H. Tang, P. Macpherson, L. S. Argetsinger, D. Cieslak, S. T. Suhr, C. Carter-Su, D. Goldman, CaM kinase IIdependent phosphorylation of myogenin contributes to activity-dependent suppression of nAChR gene expression in developing rat myotubes. Cell. Signal. 16, 551-563 (2004).

25. U. Fischer, S. Reinhardt, E. X. Albuquerque, A. Maelicke, Expression of functional alpha7 nicotinic acetylcholine receptor during mammalian muscle development and denervation. Eur. J. Neurosci. 11, 28562864 (1999).

26. J. R. Taylor, Z. Zheng, Z.-M. Wang, A. M. Payne, M. L. Messi, O. Delbono, Increased CaVbeta1 A expression with aging contributes to skeletal muscle weakness. Aging Cell. 8, 584-594 (2009). 
27. C. E. Winbanks, J. L. Chen, H. Qian, Y. Liu, B. C. Bernardo, C. Beyer, K. I. Watt, R. E. Thomson, T. Connor, B. J. Turner, J. R. McMullen, L. Larsson, S. L. McGee, C. A. Harrison, P. Gregorevic, The bone morphogenetic protein axis is a positive regulator of skeletal muscle mass. J. Cell Biol. 203, 345-357 (2013).

28. S. A. Samant, A. Kanwal, V. B. Pillai, R. Bao, M. P. Gupta, The histone deacetylase SIRT6 blocks myostatin expression and development of muscle atrophy. Sci Rep. 7, 11877 (2017).

29. T. Nakahiro, H. Kurooka, K. Mori, K. Sano, Y. Yokota, Identification of BMP-responsive elements in the mouse Id2 gene. Biochemical and Biophysical Research Communications. 399, 416-421 (2010).

30. H. Kurooka, T. Nakahiro, K. Mori, K. Sano, Y. Yokota, BMP signaling is responsible for serum-induced Id2 expression. Biochem. Biophys. Res. Commun. 420, 281-287 (2012).

31. R. Sartori, E. Schirwis, B. Blaauw, S. Bortolanza, J. Zhao, E. Enzo, A. Stantzou, E. Mouisel, L. Toniolo, A. Ferry, S. Stricker, A. L. Goldberg, S. Dupont, S. Piccolo, H. Amthor, M. Sandri, BMP signaling controls muscle mass. Nature Genetics. 45, 1309-1318 (2013).

32. V. Moresi, A. H. Williams, E. Meadows, J. M. Flynn, M. J. Potthoff, J. McAnally, J. M. Shelton, J. Backs, W. H. Klein, J. A. Richardson, R. Bassel-Duby, E. N. Olson, Myogenin and class II HDACs control neurogenic muscle atrophy by inducing E3 ubiquitin ligases. Cell. 143, 35-45 (2010).

33. Y. Li, Y. il Lee, W. J. Thompson, Changes in aging mouse neuromuscular junctions are explained by degeneration and regeneration of muscle fiber segments at the synapse. J. Neurosci. 31, 14910-14919 (2011).

34. C. R. Lamboley, V. L. Wyckelsma, T. L. Dutka, M. J. McKenna, R. M. Murphy, G. D. Lamb, Contractile properties and sarcoplasmic reticulum calcium content in type I and type II skeletal muscle fibres in active aged humans. J Physiol. 593, 2499-2514 (2015).

35. P. Balagopal, J. C. Schimke, P. Ades, D. Adey, K. S. Nair, Age effect on transcript levels and synthesis rate of muscle MHC and response to resistance exercise. Am. J. Physiol. Endocrinol. Metab. 280, E203-208 (2001).

36. K. Baar, A. R. Wende, T. E. Jones, M. Marison, L. A. Nolte, M. Chen, D. P. Kelly, J. O. Holloszy, Adaptations of skeletal muscle to exercise: rapid increase in the transcriptional coactivator PGC-1. FASEB J. 16, 1879-1886 (2002).

37. N. Brandt, M. M. Dethlefsen, J. Bangsbo, H. Pilegaard, PGC-1 $\alpha$ and exercise intensity dependent adaptations in mouse skeletal muscle. PLOS ONE. 12, e0185993 (2017).

38. R. G. Gregg, P. A. Powers, K. Hogan, Assignment of the Human Gene for the $\beta$ Subunit of the VoltageDependent Calcium Channel (CACNLB1) to Chromosome 17 Using Somatic Cell Hybrids and Linkage Mapping. Genomics. 15, 185-187 (1993).

39. K. F. Reid, R. A. Fielding, Skeletal muscle power: a critical determinant of physical functioning in older adults. Exerc Sport Sci Rev. 40, 4-12 (2012).

40. J. S. McPhee, J.-Y. Hogrel, A. B. Maier, E. Seppet, O. R. Seynnes, S. Sipilä, R. Bottinelli, Y. Barnouin, A. Y. Bijlsma, H. Gapeyeva, T. M. Maden-Wilkinson, C. G. Meskers, M. Pääsuke, E. Sillanpää, L. Stenroth, G. Butler-Browne, M. V. Narici, D. A. Jones, Physiological and functional evaluation of healthy young and older men and women: design of the European MyoAge study. Biogerontology. 14, 325-337 (2013).

41. Z. Buraei, J. Yang, The $\beta$ Subunit of Voltage-Gated Ca2+ Channels. Physiological Reviews. 90, 1461-1506 (2010).

42. T. Zhang, J. Taylor, Y. Jiang, A. S. Pereyra, M. L. Messi, Z.-M. Wang, C. Hereñú, O. Delbono, Troponin T3 regulates nuclear localization of the calcium channel Cav $\beta 1$ a subunit in skeletal muscle. Experimental Cell Research, doi:10.1016/j.yexcr.2015.05.005. 
43. S. Falcone, W. Roman, K. Hnia, V. Gache, N. Didier, J. Lainé, F. Auradé, I. Marty, I. Nishino, N. CharletBerguerand, N. B. Romero, G. Marazzi, D. Sassoon, J. Laporte, E. R. Gomes, N-WASP is required for Amphiphysin-2/BIN1-dependent nuclear positioning and triad organization in skeletal muscle and is involved in the pathophysiology of centronuclear myopathy. EMBO Molecular Medicine. 6, 1455-1475 (2014).

44. K. Takano, H. Watanabe-Takano, S. Suetsugu, S. Kurita, K. Tsujita, S. Kimura, T. Karatsu, T. Takenawa, T. Endo, Nebulin and N-WASP cooperate to cause IGF-1-induced sarcomeric actin filament formation. Science. 330, 1536-1540 (2010).

45. K. Zhao, C. Shen, Y. Lu, Z. Huang, L. Li, C. D. Rand, J. Pan, X.-D. Sun, Z. Tan, H. Wang, G. Xing, Y. Cao, G. Hu, J. Zhou, W.-C. Xiong, L. Mei, Muscle Yap Is a Regulator of Neuromuscular Junction Formation and Regeneration. J. Neurosci. 37, 3465-3477 (2017).

46. Y. Hatazawa, Y. Ono, Y. Hirose, S. Kanai, N. L. Fujii, S. Machida, I. Nishino, T. Shimizu, M. Okano, Y. Kamei, Y. Ogawa, Reduced Dnmt3a increases Gdf5 expression with suppressed satellite cell differentiation and impaired skeletal muscle regeneration. FASEB J. (2017), doi:10.1096/fj.201700573R.

47. R. Sartori, G. Milan, M. Patron, C. Mammucari, B. Blaauw, R. Abraham, M. Sandri, Smad2 and 3 transcription factors control muscle mass in adulthood. American Journal of Physiology - Cell Physiology. 296, C1248-C1257 (2009).

48. A. Bartke, Growth Hormone and Aging: Updated Review. The World Journal of Men's Health 36 (2018), doi:10.5534/wjmh.180018.

49. M. W. Hamrick, Role of the Cytokine-like Hormone Leptin in Muscle-bone Crosstalk with Aging. J Bone Metab. 24, 1-8 (2017).

50. S. D. Anker, J. E. Morley, S. von Haehling, Welcome to the ICD-10 code for sarcopenia. J Cachexia Sarcopenia Muscle. 7, 512-514 (2016).

51. V. E. Arango-Lopera, P. Arroyo, L. M. Gutiérrez-Robledo, M. U. Perez-Zepeda, M. Cesari, Mortality as an adverse outcome of sarcopenia. J Nutr Health Aging. 17, 259-262 (2013).

52. O. Delbono, Expression and Regulation of Excitation-Contraction Coupling Proteins in Aging Skeletal Muscle. Current Aging Sciencee. 4, 248-259 (2011).

53. R. J. Lopez, B. Mosca, S. Treves, M. Maj, L. Bergamelli, J. C. Calderon, C. F. Bentzinger, K. Romanino, M. N. Hall, M. A. Rüegg, O. Delbono, C. Caputo, F. Zorzato, Raptor ablation in skeletal muscle decreases Cav1.1 expression and affects the function of the excitation-contraction coupling supramolecular complex.

Biochemical Journal. 466, 123-135 (2015).

\section{Acknowledgements}

We thank Y. Marie for RNA sequencing data; N. Robil for the help in the RNA-Seq data analysis; S. Vasseur and M. Chapart for their help in collecting human muscle at the Myology Institute Myobank; V. Sazdovitch for the human spinal cord biopsy; H. Amthor and A. Stantzou for gathering negative control muscles; M. Guilbaud and F. Aurade for their expertise advices for the luciferase assay; E. Mouisel, S. Vassilopoulos, M.G. Biferi, A. Forand, E. Gargaun, P. 
Smeriglio and L. Giordani for helpful scientific discussion; D. Unni for her help as nativeEnglish reader.

\section{Funding}

This study was funded by Malakoff Médéric (to F.P.R and S.F.), Association Institute of Myology (to F.P.R and S.F.) and Association Française contre les myopathies AFM-Telethon (to F.P.R and S.F.).

\section{Author Contributions}

F.P.R. and S.F. proposed the concept, designed experiments and wrote the manuscript; S.F. and C.G. conceived and conducted in vivo experiments, cloning and GeneString design; M.T. designed and conducted experiments to validate RNAseq data presented and to demonstrate $\mathrm{h} C A C N B 1$-E expression and hCaV $\beta 1 \mathrm{E}$ localization in human muscles; C.B. performed most of qPCR and western blotting. C.G., M.T. and C.B. performed the remaining experiments and, together with S.F., analyzed the data; P.dG. performed RNAseq analysis; A.F. and M.L. conducted the force measurement; B.C. performed morphometric analysis using WEKA; S.B.Z. and L.J. provided AAV production; J.Y.H. gathered the human muscle biopsies of the European project MyoAge, maintained the blinded data, oversaw the evaluations, provided all corresponding data only after getting the results and analyzed data together with F.P.R., S.F., M.T. and C.G.; M.T. and C.G. performed separately the blinded to allocation qPCRs of MyoAge samples. No modifications, additions or exclusions were made to the validation data set from the point at which the model was locked down neither the validation data nor any subset of it had ever been used to assess or refine the model being tested.

\section{Competing Interests}

S.F. and F.P.R. hold a patent (\#18 184861.5) "Compositions for the treatment of sarcopenia or disuse atrophy". All other authors declare that they have no competing interests. Each of the authors 
confirms that this manuscript has not been previously published and is not currently under consideration by any other journal. Additionally, all of the authors have approved the contents of this paper and have agreed to the Science Translational Medicine submission policies.

\section{Data and materials availability}

All data associated with this study are available in the main text or the supplementary materials. 


\begin{tabular}{|c|c|c|c|c|c|c|}
\hline Participant & Gender & $\begin{array}{c}\text { Age } \\
\text { (years) }\end{array}$ & $\begin{array}{c}\text { Height } \\
(\mathbf{m})\end{array}$ & $\begin{array}{c}\text { Weight } \\
\mathbf{( k g )}\end{array}$ & $\begin{array}{c}\text { Lean } \\
\text { mass } \\
(\%)\end{array}$ & $\begin{array}{c}\text { Power } \\
\text { (W/Kg) }\end{array}$ \\
\hline Y1 & M & 20.9 & 1.82 & 84.4 & 82.3 & 54.7 \\
\hline Y2 & F & 24.5 & 1.63 & 56.2 & 78.4 & 51.1 \\
\hline Y3 & F & 26.1 & 1.53 & 53.0 & 70.4 & 38.4 \\
\hline Y4 & M & 26.4 & 1.76 & 56.7 & 89.2 & 48.6 \\
\hline Y5 & M & 27.1 & 1.83 & 73.8 & 75.9 & 42.4 \\
\hline Y6 & M & 37.0 & ND & ND & ND & ND \\
\hline Y7 & M & 38.0 & ND & ND & ND & ND \\
\hline Y8 & M & 42.0 & ND & ND & ND & ND \\
\hline A1 & F & 70.8 & 1.68 & 70.2 & 58.9 & 22.3 \\
\hline A2 & M & 70.9 & 1.58 & 65.1 & 70.4 & 34.5 \\
\hline A3 & M & 71.4 & 1.76 & 90.7 & 64.5 & 29.7 \\
\hline A4 & M & 71.4 & 1.68 & 84.4 & 66.3 & 27 \\
\hline A5 & F & 71.5 & 1.61 & 53.3 & 67.6 & 21.2 \\
\hline A6 & M & 71.7 & 1.67 & 69.4 & 79.2 & 35.3 \\
\hline A7 & M & 72.5 & 1.67 & 74.3 & 70.4 & 34.7 \\
\hline A8 & M & 72.9 & 1.74 & 91.6 & 64.2 & 28.1 \\
\hline A9 & M & 73.8 & 1.67 & 76.0 & 71.7 & 36 \\
\hline A10 & F & 74.2 & 1.54 & 58.3 & 64.0 & 26.3 \\
\hline A11 & M & 75.0 & 1.69 & 80.0 & 68.3 & 29.4 \\
\hline A12 & M & 76.4 & 1.58 & 67.7 & 74.7 & 25.6 \\
\hline A13 & M & 76.7 & 1.76 & 85.5 & 67.4 & 25.8 \\
\hline A14 & F & 77.8 & 1.58 & 55.5 & 70.8 & 22.2 \\
\hline A15 & M & 78.0 & 1.75 & 80.3 & 68.5 & 22.6 \\
\hline A16 & M & 79.6 & 1.67 & 67.9 & 80.9 & 26.3 \\
\hline A17 & F & 80.6 & 1.59 & 56.3 & 71.2 & 29 \\
\hline & & & & & \\
\hline & & & & & \\
\hline & & & & & \\
\hline & & & & & \\
\hline
\end{tabular}

Table 1: Characteristics of human muscles:

Young, from 20 - 42 years old (Y) and aged, from 70 - 81 years old (A) participants; gender male (M) and female (F). Lean mass (\%) was assessed by dual-energy X-ray absorptiometry. Power is expressed by Watt on kilograms (W/kg); ND: not determined. 


\section{Figure Legends}

Fig 1: Expression of an embryonic CaVß1 isoform by alternative first exon splicing after muscle denervation.

A: RT-qPCR for Cacnb1 from mouse Tibialis Anterior muscles (TAs) innervated (day zero, D0) or after the indicated number of days of denervation. Primers were designed for exons 2 and 3 of Cacnbl coding region.

B, C: (B) Western blot and (C) quantification of CaVß1 bands in innervated or denervated TAs after the indicated number of days of denervation. Actin was the loading control.

D: $\mathrm{CaV} \beta 1$ protein and Cacnbl gene and transcript variants (adapted from (41)). Cacnb1-A (NM_031173); Cacnb1-B (NM_145121); Cacnb1-C (NM_001159319); Cacnb1-D (NM_001159320); Cacnbl-E (NM_001282977); Cacnbl-F (NM_001282978). Molecular weights (MW) in brackets.

E: Cacnb1 gene showing two putative ORFs (ATG1-ATG2). Thin colored vertical lines indicate the start of transcription at ATG1 in innervated muscle and at ATG1 and ATG2 in denervated muscle.

F, G: (F) RT-PCR and (G) quantification of different Cacnbl regions in innervated (Inn) or denervated (Den) TAs validating the RNAseq data. Primers were designed for different exons (Ex) of the predicted coding region of Cacnbl. Ribosomal phosphoprotein (PO) was the loading control.

H, I: (H) RT-PCR and (I) quantification of Cacnbl-E-specific region in exon 14 and the CacnblD-specific region in exon 13 in Inn or Den TAs. PO was the loading control. 
J: RT-PCR of the expression of Cacnb1 (Ex 5-8/9) in adult TAs Inn and Den and in spinal cord (SC). PO was the loading control.

K: RT-PCR for the Cacnb1-E-specific region in exon 14 and the Cacnb1-D-specific region in exon 13 in embryonic and neonatal muscles (day 12.5 (E12.5) and 16 (E16) since fertilization; post-natal $0(\mathrm{P} 0)$ ), in adult TAs Inn or Den for 15 days. 3T3 cells were the negative control. PO was the loading control.

L: Western blot of CaV $\beta 1$ in TAs Inn or Den for 15 days and in embryonic muscle (E16; diluted 1:10) using AbCaV $\beta 1$ (central peptide). Actin was the loading control.

A, C, I: Means \pm s.e.m (A: $n=6$ mice per group; $C, I: n=3$ independent experiments $) * P<0.05$ $* * * \mathrm{P}<0.001$, (ordinary one-way Anova - Dunnett's test).

G: Means \pm s.e.m ( $\mathrm{n}=3$ independent experiments) $* * * \mathrm{P}<0.001$, (ordinary two-way - Sidak's test).

Fig 2: CaVß1E down-regulation and increased muscle atrophy by reduced GDF5 signaling after denervation.

A: RT-qPCR for Cacnb1-E (Ex 2-3) in adult TAs innervated (Inn) or denervated for 15 days (Den) treated with AAV-Sh scrambled (Scra) or AAV-ShCaV $\beta 1 E(S h C a V \beta 1 E)$.

B, C: (B) Western blot using AbCaV $\beta 1$ (central peptide) and (C) quantification of $\mathrm{CaV} \beta 1$ expression in adult TAs Inn or Den treated with Scra or ShCaVß1E. Actin was the loading control.

D, E: (D) Inn or Den muscle/body-weight ratio and (E) percentage of atrophy after denervation of adult TAs treated with Scra or ShCaV $\beta 1 E$.

F: Frequency distribution of fiber size of TAs Inn or Den treated with Scra or ShCaV $\beta 1 E$.

G-I: RT-qPCR for (G) Gdf5, (H) Gdf8 and (I) Bmp7 in Inn or Den adult TAs treated with Scra or ShCaV $\beta 1 \mathrm{E}$. 
J: Western blot (top) and quantification (bottom) of phosphorylated SMAD1/5 and SMAD5 in adult TAs Inn or Den treated with Scra or ShCaVß1E.

$\mathbf{K}, \mathbf{L}, \mathbf{M}:(\mathrm{K}, \mathrm{L})$ Immunofluorescence images and (M) quantification of TAs Inn or Den, treated with (K) Scra or (L) ShCaVß1E, stained with AchR (Magenta), SMAD4 (Yellow), Cav3 (Gray), DAPI (Cyan). Bar: $10 \mu \mathrm{m}$.

M, N: RT-qPCR for (M) $I d-1$ and (N) Id-2 in adult TAs Inn or Den treated with Scra or ShCaV $\beta 1 E$.

P: Firefly/renilla ratio in 24- or 48h-differentiated C2C12 cells co-transfected with a renilla and HSVTK-Gdf5 promoter -Luc3' with either pCDNA3-Scrambled (Scra) or pCDNA3-ShCaVß1E $(\mathrm{ShCaV} \beta 1 \mathrm{E})$.

A, C, D, G, H, I, J, M, N, O: Means \pm s.e.m. ( $\mathrm{n}=4-7$ mice per group; $\mathrm{M}: \mathrm{n}=3$ cryosections quantified), $* \mathrm{P}<0.05, * * \mathrm{P}<0.01, * * * \mathrm{P}<0.001$ (ordinary one-way Anova-Sidak’s test).

E: Means \pm s.e.m. ( $\mathrm{n}=6$ mice per group), ${ }^{*} \mathrm{P}<0.05$, (independent samples t-test (two tailed)).

F, P: Means \pm s.e.m. (F: $n=3$ cryosections quantified per condition; $O: n=3$ independent experiments), $* \mathrm{P}<0.05, * * \mathrm{P}<0.01, * * * \mathrm{P}<0.001$, (ordinary two-way Anova- F:Tukey's test; O: Sidak's test).

\section{Fig 3: Muscle mass loss during aging and alteration of CaVß1E-GDF5 axis}

A: Muscle/body-weight ratio of innervated adult TAs from 12-, 52-, 78-week-old mice.

B, C: RT-qPCR for (B) Cacnb1-E (ex 2-3) and (C) Cacnb1-D (ex13) in innervated TAs from 12, 52-, 78-week-old mice.

D-F: RT-qPCR for (D) Cacnbl (ex 2-3), (E) Cacnbl-D (ex13) and (F) Gdf5 in TAs innervated (Inn) or denervated for 15 days (Den) from mice at 12-, 52-, 78-week-old mice.

G-I: (G) Western blot using CaV $\beta 1$ (central peptide) and (H, I) quantification of CaV $\beta 1 \mathrm{E}$ and D in Inn or Den TAs from 12-, 52-, 78-week-old mice. Actin was the loading control. 
J, K: (J) Western blot and (K) quantification of phosphorylated SMAD1/5 and SMAD5 in Inn or Den TAs from 12-, 52-, 78-week-old mice.

L, M: RT-qPCR for (L) Id-1 and (M) Id2 in Inn or Den TAs from 12-, 52-, 78-week-old mice.

A, B, C: Means \pm s.e.m. (12: $n=8$ mice per group, 52: $n=9$ mice per group, $78: n=10$ mice per group) $* \mathrm{P}<0.05$, (ordinary one-way Anova-Dunnet's test).

D-F, L, M: Means \pm s.e.m. $(\mathrm{n}=6 / 7), * \mathrm{P}<0.05$, $* * \mathrm{P}<0.01$, *** $\mathrm{P}<0.001$ (ordinary one-way Anova-Sidak's test).

H, I, K: Means \pm s.e.m. ( $\mathrm{n}=3$; western blots used for quantification are showed in raw data), *P < $0.05, * * \mathrm{P}<0.01, * * * \mathrm{P}<0.001$ (ordinary one-way Anova-Sidak’s test).

Figure 4: NMJ-associated genes and MyHC expression and effect of exercise on CaVß1E expression in aged muscle

A-D: RT-qPCR for (A) Chrnal (B) Chrne (C) Chrng and (D) Musk in innervated TAs from 12-, 52-, 78-week-old mice.

E-H: RT-qPCR for (E) $M y H C-I$ (F) $M y H C$-IIA (G) $M y H C$-IIX and (H) $M y H C$-IIB in innervated TAs from 12- and 78-week-old mice.

I, J: Immunofluorescence images of TAs from (I) 12- and (J) 78-week-old mice stained with MyHC-IIA (Cyan), MyHC-IIB (Magenta) CaVß1E (Gray). MyHC-IIX are black (unstained). Bar: $25 \mu \mathrm{m}$.

K: Fluorescence intensity of CaV $\beta 1 \mathrm{E}$ staining in MyHC-IIA -IIB -IIX positive fibers in TA muscles from 12- and 78-week-old mice.

L, M: RT-qPCR for (L) Cacnb1-E and (M) Cacnb1-D in innervated TAs from 12- and 78-weekold mice in resting or exerced conditions.

N, O: RT-qPCR for (N) $M y H C I I-A$ and (O) $M y H C I I-X$ in innervated TAs from 78-week-old mice in resting or exerced conditions. 
A-D, K, L, M: Means \pm s.e.m. (A-D, L, M: n=6/8; K: MyHC-IIA n=24, MyHC-IIX n=65, MyHC-IIB $\mathrm{n}=41) * \mathrm{P}<0.05, * * * \mathrm{P}<0.001, * \mathrm{P}<0.05, * * \mathrm{P}<0.01, * * * \mathrm{P}<0.001$, (ordinary oneway Anova, A-D: Dunnett's test; K: Tukey’s test; L, M: Sidak’s test).

E-H, N, O: Means \pm s.e.m. $(\mathrm{n}=6) * \mathrm{P}<0.05$, ** $\mathrm{P}<0.01$, ***P $<0.001$, (independent samples $\mathrm{t}-$ test (two tailed)).

Fig 5: CaVß1E and GDF5 over-expression in aged muscle.

A, C, D: RT-qPCR for (A) Cacnb1-E (ex 2-3), (C) Cacnb1-D (ex13), and (D) Gdf5 in innervated TAs from 92-week-old mice treated with Scra or AAV-CaV $\beta 1 \mathrm{E}(\mathrm{CaV} \beta 1 \mathrm{E})$. A: Minimum top $\operatorname{axis}=15$.

B: Immunofluorescence images of innervated TAs from 92-week-old mice treated with Scra or

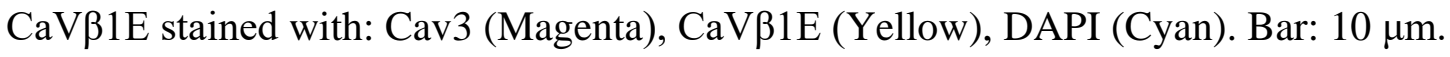

E, F: Western blot (E) and quantification $(F)$ of phosphorylated SMAD1/5 and SMAD5 in innervated TAs from 92-week-old mice treated with Scra or CaV $\beta 1 \mathrm{E}$.

G: Immunofluorescence images of innervated TAs from 92-week-old mice treated with Scra or CaVß1E stained with SMAD4 (Yellow), Cav3 (Magenta), DAPI (Cyan). Bar: $10 \mu \mathrm{m}$.

H, I: RT-qPCR for (H) $I d-1$ and (I) $I d-2$ in innervated TAs from 92-week-old mice treated with Scra or CaV $\beta 1 E$.

J: Muscle/body-weight ratio of innervated TAs from 12 and 92-week-old mice treated with Scra or from 92-week-old mice treated with $\mathrm{CaV} \beta 1 \mathrm{E}$.

K: Specific force generated by innervated TAs from 12 and 92-week-old mice treated with Scra or from 92-week-old mice treated with $\mathrm{CaV} \beta 1 \mathrm{E}$

A, C, D, H, I: Means \pm s.e.m. (Scra n=8; CaVß1E: $\mathrm{n}=8),{ }^{*} \mathrm{P}<0.05, * * \mathrm{P}<0.01, * * * \mathrm{P}<0.001$ (independent samples t-test (two tailed)).

F: Means \pm s.e.m. $(\mathrm{n}=2-3), * \mathrm{P}<0.05$, (independent samples t-test (two tailed)). 
J, K: Means \pm s.e.m. (J: Scra 12 weeks: $n=12$; Scra 92 weeks: $n=11$; CaVß1E 92 weeks n=25; GDF5 92 weeks n= 11; K: Scra 12 weeks: $n=7$; Scra 92 weeks: $n=15$; CaV $\beta 1 E 92$ weeks n= 19; GDF5 92 weeks $\mathrm{n}=6$ ), $* \mathrm{P}<0.05, * * \mathrm{P}<0.01, * * * \mathrm{P}<0.001$ (ordinary one-way Anova-Sidak's test).

\section{Fig 6: Expression of CaVß1E in human muscle: conserved compensatory mechanism?}

A: Representation of human $\mathrm{CaV} \beta 1$ protein and $\mathrm{h} C A C N B 1$ gene and transcript variants (adapted from (41)). hCACNB1-A (NM_199247); hCACNB1-B (NM_000723); hCACNB1-C

(NM_199248); predicted hCACNB1-E (XM_006722072.2). MW are in brackets.

B: RT-PCR for different isoforms of hCACNB1 in human quadriceps (Q) and fascia lata (FL1, FL2) muscle biopsies from three healthy adults. RNA from one human spinal cord (SC) biopsy was the positive control for hCACNB1-B. Primers were designed for different exons (Ex) of the coding region of hCACNB1. Human Ribosomal phosphoprotein (hPO) is the loading control. C: RT-PCR for hCACNB1-E in human Q, FL1, FL2 muscle biopsies from three healthy adults. RNA from SC biopsy was the positive control for the expression of exon 14 of hCACNB1-B. Primers were designed for different exons (Ex) of the coding region of hCACNB1. hPO was the loading control.

D: Western blot using CaVß1 (central peptide) in human FL1 and FL2 muscle biopsies from the same adult healthy subjects as in B and C. Actin was the loading control.

E: Hematoxylin/eosin (H/E) and immunofluorescence images of human FL1 and FL2 muscle used in B, C and D stained with hCaVß1E (Yellow), Cav3 (Magenta), DAPI (Cyan). Bar H/E: 50 $\mu \mathrm{m}$; bar IF: $10 \mu \mathrm{m}$.

F, G: Distribution of $(\mathrm{F})$ lean mass percentage and $(\mathrm{G})$ Power in human quadriceps biopsies from healthy young and aged volunteers (Table 1). 
H, I: Distribution of $(\mathrm{H}) \mathrm{h} C A C N B$ l-E or (I) hCACNB1-A expression in human quadriceps biopsies from healthy young and aged volunteers (Table 1).

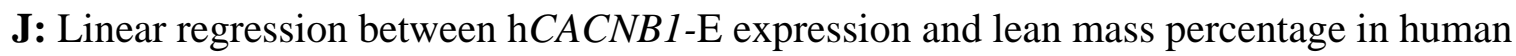
quadriceps biopsies from healthy young and aged volunteers, (Table 1).

K: Distribution of hGDF5 (pink triangles, left y axis) and hCACNB1-E (blue circles, right y axis) expression in human quadriceps biopsies from healthy aged volunteers (Table 1) having increasing lean mass percentage. Dot black line indicates the average of lean mass percentage of the young group.

F-I: Means \pm s.e.m. (Young: $\mathrm{n}=8$; Aged: $\mathrm{n}=17$ ) $* * \mathrm{P}<0.01$, ***P $<0.001$, (independent-samples t-test (two-tailed)). 